\title{
LA ORGANIZACIÓN DE LA INSPECCIÓN DE EDUCACIÓN EN ESPAÑA
}

\section{The organization of Educational Inspection in Spain}

\author{
María Mercé Berengueras Pont \\ Inspección de Educación de Barcelona \\ Correo-e: mberenpont@gmail.com \\ José María Vera Mur \\ Inspección de Educación de Lleida \\ Correo-e: josemaria.vera@gmail.com \\ Recibido: I de junio de 2018 \\ Envío a informantes: II de junio de 2018 \\ Aceptación definitiva: 22 de julio de 2018
}

Resumen: El artículo analiza la Inspección de Educación en España, en el actual contexto normativo, organizativo y funcional, sin obviar, en su introducción, sus orígenes y antecedentes históricos próximos.

Se analiza de manera detallada el actual marco normativo, legal y reglamentario, tanto el de carácter básico como el específico desarrollado hasta el momento por el propio Ministerio de Educación y las comunidades autónomas.

Las funciones y atribuciones básicas y específicas de la Inspección, sus componentes, su organización y dependencia dentro de la estructura organizativa de la Administración Educativa, junto con el papel de cambio de la Inspección, completan el contenido del artículo.

Palabras clave: Administración educativa; Inspección educativa; Inspección escolar; Supervisión escolar; Supervisión educativa.

Aвsтract: The article analyzes the Educational Inspection in Spain, in the current normative, organizational and functional context, without forgetting in its introduction, its origins and close historical background.

The current normative, legal and regulatory framework is analyzed in detail, both the basic and the specific character developed so far by the Ministry of Education and the Autonomous Communities. 
The functions and basic and specific attributions of the Inspection, its components, its organization and dependence within the organizational structure of the Educational Administration, together with the inspection change role, complete the content of the article.

KeY words: Educational Administration; Educational Inspection; School Inspection; School Supervision; Educational Supervision.

\section{Introducción}

$\mathrm{L}$

A ACtual Inspección de Educación en España se remonta a la Ley de Instrucción Pública de 9 de septiembre de 1857 (más conocida como la Ley Moyano), cuando en su artículo 294 establece que «el Gobierno ejercerá su inspección y vigilancia sobre los establecimientos de instrucción, así públicos como privados».

Se inicia con la Inspección de Enseñanza Primaria y tiene sus antecedentes reglamentarios reguladores en una disposición que data del 20 de mayo de i849, primer reglamento de la Inspección de Enseñanza Primaria, promulgada unas semanas después de su creación por el Real Decreto de 30 de marzo de i849, publicado en la Gaceta de Madrid (precedente del actual $B O E$ ) número 5315 de 2 de abril, seguida de un segundo reglamento, el de 27 de marzo de 1896 , al que siguieron una serie de disposiciones reglamentarias, todas ellas referidas a la Inspección de Enseñanza Primaria, la tercera de 2 de diciembre de 1932 y la cuarta de 3 de noviembre de 1967.

En lo que respecta a las Enseñanzas Medias (actuales Secundaria y Bachillerato), la Ley de 20 de septiembre de 1938 estableció en su base XI con carácter permanente la Inspección de Enseñanza Media, pero no es hasta la Ley de Ordenación de las Enseñanzas Medias de 26 de febrero de 1953, cuando se estableció con carácter técnico y estable la Inspección, siendo desarrollada su constitución y funcionamiento en el Decreto de 5 de mayo de I954, completado más tarde con el Decreto 898/1963 de 25 de abril, que la regulaba orgánicamente.

La Ley 3I/I980, de 2I de junio, creó un tercer cuerpo de inspectores, el Cuerpo Especial de Inspectores Técnicos de Formación Profesional, que desarrolló su regulación, organización y funcionamiento mediante el Real Decreto 657/1982 de i7 de marzo.

No obstante, la profesionalización de la Inspección Educativa se debe a la Ley General de Educación (LGE) de I970, que reforzó los distintos cuerpos de inspectores, tanto de enseñanza general básica como de enseñanza media, dejando la puerta abierta a una inspección de la formación profesional.

El Decreto 664/1973, de 22 de marzo, sobre funciones del Servicio de Inspección Técnica de Educación, y el Real Decreto 657/1982, de 17 de marzo, regulador de la Inspección Técnica del Estado de Formación Profesional, nos confirman la existencia de inspecciones nivelares hasta las últimas décadas del siglo pasado.

Tras la Constitución de 1978, en el Estado de las autonomías, se inicia una nueva etapa. La Carta Magna establece que «los poderes públicos inspeccionarán y homologarán el sistema educativo para garantizar el cumplimiento de las leyes» (art. 27.8). Este mandato favoreció que en 1984 se definiese un nuevo marco legal para la Inspección educativa con el establecimiento de un sistema de acceso distinto y la extinción de los cuerpos existentes hasta el momento. 
LA ORGANIZACIÓN DE LA INSPECCIÓN DE EDUCACIÓN EN ESPAÑA

Con la Ley 30/1984, en su disposición adicional decimoquinta se crea el nuevo Cuerpo de Inspectores al Servicio de la Administración Educativa (CISAE). En él se integran el Cuerpo de Inspectores de Educación Básica, el de Inspectores de Bachillerato y el de Inspectores Técnicos de Formación Profesional, con los efectivos en ese momento de los cuerpos que se suprimen, quedando amortizadas las vacantes que en lo sucesivo se produzcan.

Posteriormente, con el desarrollo legislativo del sistema educativo se volvió a dar un nuevo enfoque de la Inspección, con la publicación de la actualmente derogada LOPEG (Ley Orgánica 9/1995, de 20 de noviembre, de la participación, la evaluación y el gobierno de los centros docentes), que supuso el retorno del Cuerpo de Inspectores de Educación, que la disposición adicional quince, apartado siete de la Ley 30/1984, de 2 de agosto, de medidas para la Reforma de la Función Pública, había suprimido, y con ello la consiguiente modificación del sistema de acceso, selección y provisión de puestos de trabajo, que tendría su proyección en las Administraciones Educativas.

La LOCE (Ley Orgánica Io/2002 de 23 de diciembre, de calidad de la educación), actualmente también derogada, vuelve a regular los aspectos relacionados con las funciones, la organización y la formación de la Inspección de Educación, siendo este último aspecto el más novedoso respecto a las anteriores disposiciones legales reguladoras.

Actualmente, la LOE (Ley Orgánica 2/2006, de 3 de mayo, de educación), modificada por la LOMCE (Ley Orgánica 8/20I3, de 9 de diciembre, para la mejora de la calidad educativa), considera la Inspección como la garante del cumplimiento de las leyes, de los derechos y de la observancia de los deberes de cuantos participan en los procesos de enseñanza y aprendizaje, así como en la mejora del sistema educativo y la calidad y equidad de la enseñanza.

\section{Actual contexto normativo de la Inspección de Educación}

El marco jurídico de la Inspección de Educación y, en especial, en lo que concierne a sus funciones, tiene una doble vertiente, una de carácter general como elemento integrante de la Administración y como funcionarios públicos y otra más específica como Inspección de Educación.

Como elemento integrante de la Administración, la Inspección de Educación forma parte del Ministerio y de las Consejerías o Departamentos de Educación de las Comunidades Autónomas respectivas y, por tanto, de la Administración Educativa, tanto a nivel central como periférico a través de las direcciones provinciales, delegaciones o servicios territoriales. De ahí que sus funciones propias de desarrollen respetando el marco jurídico de actuación general de las administraciones públicas, es decir, el artículo io3.I. de la Constitución, las Leyes 39/2015 y 40/2015 de I de octubre, del procedimiento administrativo común de las administraciones públicas y del régimen jurídico del sector público, respectivamente, y, en su caso, de las leyes de procedimiento administrativo de las Comunidades Autónomas.

El artículo I03.I. de la Constitución regula la actuación de las administraciones públicas en el sentido de que estas servirán con objetividad a los intereses generales y actuarán de acuerdo con los principios de eficacia, jerarquía, descentralización, desconcentración y coordinación, con sumisión plena a la Ley y al Derecho. Las Leyes 
39/2015 y 40/20I5 regulan las bases del régimen jurídico, el procedimiento administrativo común y el sistema de responsabilidades de las administraciones públicas, por lo que las actuaciones de los inspectores de educación en el desarrollo de sus funciones específicas han de tener en cuenta los preceptos regulados en la misma, especialmente cuando las actuaciones tengan un carácter administrativo.

Como funcionarios públicos, las funciones atribuidas a la Inspección de Educación son ejercidas por el Cuerpo de Inspectores al Servicio de la Administración Educativa (CISAE) en vía de extinción por amortización de plazas y por el actual Cuerpo de Inspectores de Educación (CIE) y, por tanto, cuando desarrollen sus funciones específicas, han de tener en cuenta el marco jurídico que determina la legislación reguladora de la función pública, entendida esta como la propia del territorio de las respectivas administraciones educativas. De acuerdo con el sentido de las leyes de función pública respectivas, los inspectores han de actuar en todo momento con diligencia, profesionalidad, imparcialidad y sometimiento a la Constitución, al Estatuto de Autonomía respectivo, las leyes, los reglamentos que los complementan y el derecho en general, teniendo siempre presentes los principios de legalidad, objetividad, economía, eficacia y eficiencia.

Actualmente, además del artículo 27.8. de la Constitución, el fundamento normativo de carácter legal de la Inspección de Educación reside en la LOE, modificada por la LOMCE (Ley Orgánica 8/20I3, de 9 de diciembre, para la mejora de la calidad educativa) en sus artículos I5I al I54 y en disposiciones adicionales y transitoria:

Referencias a la Inspección de Educación en la LOE

\begin{tabular}{|l|l|}
\hline $\begin{array}{l}\text { Título viI - Capítulo II } \\
\text { artículos I5I al I54) }\end{array}$ & $\begin{array}{l}\text { - funciones } \\
\text { - atribuciones } \\
\text { - componentes } \\
\text { - organización }\end{array}$ \\
\hline $\begin{array}{l}\text { Disposición adicional } \\
\text { décima, } 5\end{array}$ & $\begin{array}{l}\text { - requisitos para acceder al Cuerpo de Inspectores de Educación } \\
\text { - cuerpo de origen, experiencia docente previa, titulaciones, conocimiento lengua cooficial }\end{array}$ \\
\hline $\begin{array}{l}\text { Disposición adicional } \\
\text { duodécima, } 4\end{array}$ & $\begin{array}{l}\text { - criterios aplicables en el concurso-oposición } \\
\text { - fases de oposición y concurso } \\
\text { - reserva de plazas para concurso de méritos destinado a profesores con evaluación positiva en } \\
\text { el cargo de director }\end{array}$ \\
\hline $\begin{array}{l}\text { Disposición adicional } \\
\text { decimotercera }\end{array}$ & $\begin{array}{l}\text { - desempeño de la función inspectora por funcionarios no pertenecientes al Cuerpo de } \\
\text { Inspectores de Educación } \\
\text { - continuación con carácter definitivo hasta su jubilación }\end{array}$ \\
\hline $\begin{array}{l}\text { Disposición transitoria } \\
\text { segunda }\end{array}$ & - jubilación voluntaria anticipada en iguales condiciones que el profesorado \\
\hline
\end{tabular}

Fuente: LOE.

En aquellas comunidades autónomas que han publicado su Ley de Educación, seis en la actualidad, también la Inspección de Educación está presente en su articulado, por lo que se puede considerar otro fundamento normativo de carácter legal de la misma.

- Andalucía, artículos I45 al I50 de la Ley I7/2007 de io de diciembre.

- Canarias, artículo i8 de la Ley 6/20I4 de 25 de julio.

- Cantabria, artículos 152 al I57 de la Ley 6/2008 de 26 de diciembre.

- Castilla-La Mancha, artículos I62 al I64 de la Ley 7/2010 de 20 de julio). 
LA ORGANIZACIÓN DE LA INSPECCIÓN DE EDUCACIÓN EN ESPAÑA

- Cataluña, artículos I77 al i8I de la Ley i2/2009 de io de julio.

- Extremadura, artículos I8I al i86 de la Ley 4/20II de 7 de marzo.

Sin aparecer en su título como ley de educación, el País Vasco también dedica un artículo, el veinticinco, en su Ley i/1993, de I9 de febrero, de la Escuela Pública Vasca a la Inspección de Educación.

Del estudio comparativo de las leyes de educación autonómicas, se pueden considerar algunas diferencias en sus aspectos reguladores, señalando como más significativas las siguientes:

- La exclusiva remisión de las funciones y atribuciones al marco legal estatal de la LOE, sin establecer ninguna propia, es el caso de las leyes de Andalucía y Castilla-La Mancha.

- Regulación muy específica y diferenciada de la formación y evaluación de los inspectores de educación, llegando a determinar los aspectos que deberá favorecer el funcionamiento de la propia Inspección, es el caso de la ley de Cantabria.

- Los tres aspectos diferenciadores que aporta la ley de Cataluña:

- Adecuación de la implementación de las funciones y atribuciones de la Inspección al régimen de autonomía de los centros.

- Concreción de la provisión de los puestos de trabajo en comisión de servicios y la referencia a su situación administrativa.

- Creación de un cuerpo propio de Inspectores de Educación.

Al margen de la normativa legal mencionada, la Inspección de Educación figura también como destinataria de funciones y atribuciones de carácter específico, como colaboradora en diversas actuaciones de las Administraciones Públicas, como es el caso a modo de ejemplo de la Ley Orgánica I/2004, de 28 de diciembre, de medidas de protección integral contra la violencia de género, en su artículo 9, "Los servicios de Inspección Educativa velarán por el cumplimiento y aplicación de los principios y valores recogidos en este capítulo en el sistema educativo destinados a fomentar la igualdad real entre mujeres y hombres».

A nivel reglamentario, las Administraciones Educativas competentes en materia educativa, incluido el propio Ministerio de Educación, también han regulado sus servicios de Inspección de Educación, existiendo actualmente vigentes las siguientes reglamentaciones:

- Ministerio de Educación: Orden de 29 de febrero de 1996, por la que se regula la organización y el funcionamiento de la Inspección ( $B O E$ del 2 de marzo).

- Andalucía: Decreto II5/2002, de 25 de marzo, por el que se regula la organización y el funcionamiento de la Inspección Educativa ( $B O J A$ del 30 ).

- Aragón: Decreto 32/2018, de 20 de febrero, por el que se regula la Inspección de Educación en la Comunidad Autónoma de Aragón ( $B O A$ del I de marzo).

- Asturias: Resolución de I de agosto de 20r2, de la Consejería de Educación, por la que se aprueban las instrucciones de organización y funcionamiento del Servicio de Inspección Educativa (BOPA del I4); el art 30 del Decreto 65/2015, de 13 de agosto, que regula la organización básica de la Consejería de Educación (BOPA del I4) regula sus funciones.

- Baleares: Decreto 36/200I, de 9 de marzo, por el cual se regula la Inspección Educativa en el ámbito de la enseñanza no universitaria ( 
- Canarias: Decreto 52/2009, de 12 de mayo, por el que se aprueba el Reglamento de Ordenación de la Inspección de Educación de la Comunidad Autónoma de Canarias (BOIC del 22).

- Cantabria: Orden ECD/III/20I5, de 30 de septiembre, por la que se regula la organización y funcionamiento de la Inspección Educativa de la Comunidad Autónoma de Cantabria ( $B O C$ del 8 de octubre), modificada por la Orden ECD/I26/20I7, de 25 de octubre ( $B O C$ del 2 de noviembre).

- Castilla-La Mancha: Decreto 34/2008, de 26 de febrero, por el que se establece la ordenación de la Inspección de Educación de Castilla-La Mancha (DOCM del 29), desarrollado por la Orden de 8 de abril de 2008 (DOCM del I8).

- Castilla y León: Decreto 92/2004, de 29 de julio, por el que se regula la Inspección Educativa en Castilla y León ( $B O C y L$ del 3 de agosto), desarrollado por la Orden EDU/1373/2008 de 23 de julio (BOCyL del 30).

- Cataluña: Decreto 266/200o de I de julio, por el que se regula la Inspección en Cataluña (DOGC del 8 de agosto) modificado por el Decreto I48/2002, de 22 de mayo (DOGC del 7 de junio), desarrollados por las Órdenes ENs/289/2002, de 3I de julio y ENS/385/2002, de I3 de noviembre (DOGC del I2 de agosto y del 2I de noviembre respectivamente).

- Extremadura: Orden de 29 de febrero de 1996, por la que se regula la organización y el funcionamiento de la Inspección ( $B O E$ del 2 de marzo) con carácter supletorio al no tener legislación propia autonómica. Para el trienio 2017-2020, la Instrucción del Secretario General de Educación de 17 de julio de 20I7, regula la organización y el funcionamiento y el Plan Director de la actuación de la Inspección.

- Galicia: Decreto 99/2004, de 2r de mayo, por el que se regula la organización y el funcionamiento de la Inspección Educativa y el acceso al Cuerpo de Inspectores de Educación en Galicia ( $D O G$ del 25).

- Madrid: Resolución de 20 de abril de 2007 de la Viceconsejería de Educación, por la que se dictan instrucciones de organización y funcionamiento de la Inspección Educativa de la Comunidad de Madrid (вОСм del I7 de mayo).

- Murcia: Decreto 316/2015, de 29 de diciembre, por el que se ordena y regula la Inspección de Educación en la Comunidad Autónoma de la Región de Murcia (BORM del 3I), desarrollado por la Orden de i2 de julio de 2017 (BORM del 25).

- Navarra: Decreto Foral 80/2008, de 30 de junio, por el que se regula la organización y el funcionamiento de la Inspección Educativa del Departamento de Educación (BON del 4 de agosto).

- País Vasco: Decreto 98/2016, de 28 de junio, de la Inspección de Educación en la Comunidad Autónoma del País Vasco ( $B O P V$ del 4 de julio).

- La Rioja: Decreto 3/2010, de 22 de enero, por el que se regula la organización y funcionamiento de la Inspección Técnica Educativa de la Comunidad Autónoma de La Rioja (BOLR del 27).

- Valencia: Decreto 80/2017, de 23 de junio, por el que se regula la actuación, el funcionamiento y la organización de la Inspección de Educación de la Comunidad Valenciana (DOGV del 5 de julio). 


\section{Funciones y atribuciones de la Inspección}

De acuerdo con el artículo I5i de la LOE, las funciones de la Inspección Educativa, entendidas estas como las actividades particulares y específicas de cada órgano administrativo, afectan los ámbitos de supervisión y control, evaluación, asesoramiento, orientación e información y quedan determinadas de la siguiente manera:

Funciones y atribuciones de la Inspección

\begin{tabular}{|l|l|}
\hline $\begin{array}{l}\text { Supervisión } \\
\text { y control }\end{array}$ & $\begin{array}{l}\text { - Supervisar y controlar, desde el punto de vista pedagógico y organizativo, el funcionamiento de los } \\
\text { centros educativos, así como los programas que en ellos inciden. } \\
\text { - Supervisar la práctica docente, la función directiva y colaborar en su mejora continua. } \\
\text { - Velar por el cumplimiento, en los centros educativos, de las leyes, reglamentos y demás disposiciones } \\
\text { vigentes que afecten al sistema educativo. } \\
\text { - Velar por el cumplimiento y aplicación de los principios y valores recogidos en esta Ley, incluidos los } \\
\text { destinados a fomentar la igualdad real entre hombres y mujeres. }\end{array}$ \\
\hline Evaluación & - Participar en la evaluación del sistema educativo y de los elementos que lo integran. \\
\hline Asesoramiento & $\begin{array}{l}\text { - Asesorar, orientar e informar a los distintos sectores de la comunidad educativa en el ejercicio de sus } \\
\text { derechos y en el cumplimiento de sus obligaciones. }\end{array}$ \\
\hline $\begin{array}{l}\text { Orientación } \\
\text { e información }\end{array}$ & $\begin{array}{l}\text { - Emitir los informes solicitados por las Administraciones Educativas respectivas o que se deriven del } \\
\text { conocimiento de la realidad propio de la Inspección Educativa, a través de los cauces reglamentarios. }\end{array}$ \\
\hline
\end{tabular}

Fuente: LOE, artículo I5I.

Estas se complementan, en algunos casos, con las establecidas en las Leyes de Educación autonómicas en vigor y en los reglamentos reguladores de cada una de las Administraciones Educativas competentes.

En cuanto a las atribuciones, entendidas como las facultades o poderes de una persona por razón de su cargo o función, el artículo 53 del texto consolidado de la LOE establece como básicas las siguientes:

I. Conocer directamente todas las actividades que se realicen en los centros, a los que tendrán libre acceso.

2. Examinar y comprobar la documentación académica, pedagógica y administrativa de los centros.

3. Recibir de los restantes funcionarios y responsables de los centros y servicios educativos, públicos y privados, la necesaria colaboración para el desarrollo de sus actividades, para cuyo ejercicio los inspectores tendrán la consideración de autoridad pública.

4. Cualesquiera otras que le sean atribuidas por las Administraciones Educativas, dentro del ámbito de sus competencias.

Como en las funciones, en algunos casos se complementan las atribuciones mencionadas con las establecidas en las Leyes de Educación autonómicas en vigor y en los reglamentos reguladores de cada una de las Administraciones Educativas competentes.

\section{Componentes}

- Funcionarios del Cuerpo de Inspectores de Educación (CIE).

- Funcionarios de cuerpos docentes en comisión de servicios como inspectores accidentales. 
El artículo I52 de la LOE determina que la Inspección Educativa será ejercida por las Administraciones Educativas a través de funcionarios y funcionarias públicos del Cuerpo de Inspectores de Educación (CIE), así como los pertenecientes al extinguido Cuerpo de Inspectores al Servicio de la Administración Educativa (CISAE) creado por la Ley 30/1984, de 2 de agosto, de Medidas para la Reforma de la Función Pública, modificada por la Ley 23/1988, de 28 de julio, que no hubieran optado en su momento por su incorporación al de Inspectores de Educación (CIE). Ambos cuerpos tienen carácter de cuerpo docente clasificado en el grupo A de los que establece el artículo 25 de la Ley 30/1984, de 2 de agosto, de medidas para la Reforma de la Función Pública.

También podrán ejercer función inspectora educativa, aquellos funcionarios y funcionarias de los cuerpos docentes que accedieron a la función inspectora de conformidad con las disposiciones de la Ley $30 / 1984$, de 2 de agosto, de Medidas para la Reforma de la Función Pública, modificada por la Ley 23/1988, de 28 de julio, y que no hubieran accedido al Cuerpo de Inspectores de Educación a la entrada en vigor de la LOE, los cuales, de acuerdo con la disposición adicional decimotercera, apartado 2, podrán continuar desempeñando la función inspectora con carácter definitivo y hasta su jubilación como funcionarios o funcionarias, de conformidad con las disposiciones por las que accedieron al mismo.

\section{Requisitos para acceder}

La LOE establece en el apartado 5 de la disposición adicional décima, de manera expresa y diferenciada a la anterior ley orgánica, como requisitos para acceder al Cuerpo de Inspectores de Educación, además de pertenecer a alguno de los cuerpos que integran la función pública docente, como ya lo hiciera la LOCE, los de poseer el título de doctor, licenciado, ingeniero, arquitecto o titulación equivalente y acreditar el conocimiento de la lengua cooficial de la Comunidad Autónoma de destino, de acuerdo con su normativa.

Así mismo las personas aspirantes deberán contar con una antigüedad mínima de seis (6) años en alguno de los cuerpos de la función pública docente y una experiencia docente de igual duración.

\section{Forma de acceso}

A partir de la entrada en vigor de la LOE, según el apartado cuarto de su disposición adicional duodécima la forma de acceso al Cuerpo de Inspectores de Educación queda de la siguiente manera:

- El acceso se realizará mediante concurso-oposición.

- El proceso constará de tres fases:

- Fase de concurso, dónde se valorará la trayectoria profesional de las personas candidatas y sus méritos específicos como docentes, el desempeño de cargos directivos con evaluación positiva y la pertenencia a alguno de los cuerpos de catedráticos de la función pública docente.

- Fase de oposición, consistente en una prueba en la que se valorarán los conocimientos pedagógicos, de administración y legislación educativa de las personas 
LA ORGANIZACIÓN DE LA INSPECCIÓN DE EDUCACIÓN EN ESPAÑA MARÍA MERCÉ BERENGUERAS PONT Y JOSÉ MARÍA VERA MUR

aspirantes adecuada a la función inspectora que van a realizar, así como los conocimientos y técnicas específicos para el desempeño de la misma.

Consta de tres partes:

I. Desarrollo por escrito de un tema referido a la parte A del temario, elegido por el aspirante de entre dos extraídos por sorteo por el tribunal.

2. Exposición oral de un tema referido a la parte B del temario, elegido por el aspirante de entre dos extraídos por sorteo por el tribunal.

3. Análisis de un caso práctico sobre técnicas adecuadas para la actuación de la Inspección de Educación propuesto por el tribunal.

- Fase de prácticas, consistente en realizar un período de prácticas de carácter selectivo, una vez seleccionadas las personas candidatas, al final de la cual serán nombrados/as funcionarios/as de carrera del Cuerpo de Inspectores de Educación.

- En las convocatorias de acceso al Cuerpo de Inspectores de Educación, las Administraciones Educativas podrán reservar hasta un tercio de las plazas para la provisión mediante concurso de méritos destinado a los profesores/as que, reuniendo los requisitos generales, hayan ejercido con evaluación positiva, al menos durante tres mandatos, el cargo de director o directora.

Desde el día 22 de diciembre de 2009 los temas vigentes para el acceso al Cuerpo de Inspectores de Educación están establecidos por la Orden EDU/3429/2009, de II de diciembre, publicada en el BOE. 2I.I2.2009, que determina dos partes claramente diferenciadas en la fase de oposición:

Parte $A$, que consta de cincuenta y cinco (55) temas, que figuran relacionados en el anexo I de la Orden. Incluyen temas generales relativos a cuestiones pedagógicas sobre organización curricular, organización escolar, gestión de centros educativos, administración y legislación educativa básica, así como funciones inspectoras de control, evaluación y asesoramiento.

Parte $B$, que consta de veintiún (2I) temas, que figuran relacionados en el anexo II de la Orden. Incluyen temas de carácter específico referidos a las características propias de los niveles y etapas educativas, al desarrollo curricular y a la correspondiente metodología didáctica y a la organización y administración de los centros y a la legislación propia de la Administración Educativa convocante. A estos temas, las Administraciones Educativas correspondientes podrán añadir hasta un máximo de 20 temas más.

\section{Organización y estructura}

La principal novedad se da en considerar un desarrollo reglamentario de sentido más amplio para las Administraciones Educativas competentes, encargando a estas la regulación de la organización y funcionamiento de la Inspección Educativa sin limitaciones de reglamentaciones previas de carácter básico.

También la organización de los perfiles profesionales de los inspectores y de las inspectoras en base a unos criterios preestablecidos es otro de los aspectos novedosos a tener en cuenta, así como su consideración de mérito en los procedimientos para la provisión de puestos de trabajo. 
El artículo I54 de la LOE, considera:

I. Las Administraciones Educativas regularán la estructura y el funcionamiento de los órganos que establezcan para el desempeño de la Inspección Educativa en sus respectivos ámbitos territoriales.

2. La estructura organizativa podrá organizarse sobre la base de los perfiles profesionales de los inspectores, entendidos en función de los criterios siguientes: titulaciones universitarias, cursos de formación en el ejercicio de la Inspección, experiencia profesional en la docencia y experiencia en la propia Inspección Educativa.

3. En los procedimientos para la provisión de puestos de trabajo en la Inspección Educativa podrán tenerse en consideración las necesidades de las respectivas Administraciones Educativas y podrá ser valorada como mérito la especialización de las personas aspirantes de acuerdo con las condiciones descritas para organizar los perfiles profesionales.

La LOE no regula de manera expresa los aspectos de la formación de la Inspección Educativa, ni tampoco hace referencia a unas especialidades de la inspección de carácter básico, como lo planteaba la anterior ley orgánica, por lo que es de prever que estos aspectos serán desarrollados reglamentariamente por las Administraciones Educativas competentes, a través de sus respectivos decretos reguladores de la Inspección Educativa.

Hasta una nueva regulación reglamentaria por parte de las Administraciones Educativas correspondientes la dependencia de la Inspección de Educación en cada una de ellas es la siguiente:

Organización de la Inspección de Educación en España

\begin{tabular}{|l|l|}
\hline Ministerio de Educación & - Dirección General de Evaluación y Cooperación Territorial \\
\hline Andalucía & - Viceconsejería de Educación \\
\hline Aragón & - Secretaría General Técnica \\
\hline Asturias & - Directamente del Consejero de Educación, como Servicio de Inspección Educativa \\
\hline Baleares & - Dirección General de Formación Profesional y de Inspección Educativa \\
\hline Canarias & - Viceconsejería de Educación \\
\hline Cantabria & - Dirección General de Innovación y Centros Educativos, como Servicio de Inspección \\
\hline Castilla-La Mancha & - Dirección General de Coordinación y Política Educativa \\
\hline Castilla y León & - Dirección General de Política Educativa Escolar \\
\hline Cataluña & - Secretaría de Políticas Educativas \\
\hline Extremadura & - Secretaría General de Educación \\
\hline Galicia & - Dirección General de Centros y Ordenación Educativa \\
\hline Comunidad de Madrid & - Viceconsejería de Educación \\
\hline Murcia & - Secretaría General \\
\hline Comunidad Foral de Navarra & - Dirección General de Educación, Formación Profesional y Universidades \\
\hline País Vasco & - Viceconsejería de Educación \\
\hline La Rioja & - Consejero/a de Educación, como Servicio de Inspección \\
\hline Comunidad Valenciana & - Secretaría Autonómica de Educación \\
\hline
\end{tabular}

Fuente: LOE y reglamentos autonómicos de la regulación de la Inspección. 
La organización de la Inspección de Educación, de acuerdo con los reglamentos e instrucciones de organización y funcionamiento reguladores publicados y vigentes hasta la fechas es la siguiente:

Estructura organizativa y funcional de la Inspección de Educación en España

\begin{tabular}{|c|c|c|c|c|c|}
\hline \multirow[b]{2}{*}{$\begin{array}{c}\text { Administración } \\
\text { Educativa }\end{array}$} & \multicolumn{2}{|c|}{ Cargos territoriales } & \multirow[b]{2}{*}{$\begin{array}{c}\text { Demarcación } \\
\text { territorial }\end{array}$} & \multirow[b]{2}{*}{$\begin{array}{c}\text { Organización } \\
\text { del trabajo }\end{array}$} & \multirow[b]{2}{*}{ Tiempo de adscripción } \\
\hline & $\begin{array}{c}\text { Inspector } \\
\text { jefe }\end{array}$ & $\begin{array}{l}\text { Inspector } \\
\text { jefe } \\
\text { adjunto }\end{array}$ & & & \\
\hline Ministerio de Educación & Sí & Sí & distrito & grupos de trabajo & máx. 6 años \\
\hline Andalucía & Sí & Sí & zonas & áreas & 5 años \\
\hline Aragón & Sí & Sí & distrito & áreas específicas & 4 años \\
\hline Asturias & Sí & Sí & distritos & áreas & 3-5 años \\
\hline Islas Baleares & Sí & & demarcación & áreas específicas & \\
\hline Canarias & Sí & Sí & zonas & equipos de trabajo & 4 años \\
\hline Cantabria & Sí & Sí & distrito & áreas específicas & 3-5 años \\
\hline Castilla-La Mancha & Sí & Sí & zonas & áreas específicas & 4 años \\
\hline Castilla y León & Sí & & distrito & grupos de trabajo & 5 años \\
\hline Cataluña & Sí & Sí & áreas geográficas & áreas y ámbitos & 5 años \\
\hline Extremadura & Sí & & distrito & & \\
\hline Galicia & Sí & & sector y subsectores & equipos de trabajo & 5 años \\
\hline Comunidad de Madrid & Sí & Sí $(*)$ & distritos & & 4 años \\
\hline Murcia & Sí & Sí & distritos & áreas de trabajo & 4 años (máx. 8 en el distrito) \\
\hline Navarra & Sí & & zonas & áreas específicas & 3-5 años \\
\hline País Vasco & Sí & $(* *)$ & $\begin{array}{l}\text { zonas } \\
\text { circunscripción }\end{array}$ & grupos de trabajo & 6 años \\
\hline La Rioja & Sí & & zonas & áreas específicas & 4 años \\
\hline Valencia & Sí & $(* * *)$ & circunscripción & áreas específicas & 4 años \\
\hline
\end{tabular}

(*) Si el volumen del trabajo lo aconseja.

(*) En casos de ausencia actúa de inspector jefe uno de los inspectores jefes de zona territorial.

(***) Solo figura el cargo de inspector secretario.

Fuente: LOE y reglamentos autonómicos de la regulación de la Inspección.

\section{Bibliografía}

Berengueras Pont, M. y Vera Mur, J. M. a (2015) La Inspección de Educación en España: Actual Contexto Normativo, Organizativo y Funcional. Revista Iberoamericana sobre Calidad, Eficacia y Cambio en Educación-REICE, 2015, I3 (4), II3-I29.

Esteban, S. (2007) Reflexiones sobre las antinomias de la Inspección Educativa en España. Un problema sin resolver. Avances de Supervisión Educativa, 6. Recuperado el 20 de marzo de 2015 , de http://www.adide.org/revista/index.php?option=com_content\&task=view\&i$\mathrm{d}=204 \&$ Itemid $=47$.

Iniesta, A.; Alhambra, C. y Cirac, V. (1999) La Inspección en la Educación Secundaria. Revista de Educación, 320, 39-59.

López del Castillo, M. T. (2000) La inspección del Bachillerato en España (1845-1984). Madrid: UNED. 
López JoRDAN, L. J. (20I7) Estudio de la situación de la Inspección de Educación en las Comunidades Autónomas. Revista Supervisión, 21, núm. 46, octubre 2017.

Martínez, C. y Hernández, V. (2015) La evaluación de la Supervisión Educativa en las condiciones actuales. Revista Iberoamericana sobre Calidad, Eficacia y Cambio en Educación-REICE, I3 (2), 85-98.

Mayorga, A. (1999) La Inspección en el nivel de la Educación Primaria. Proceso histórico. Revista de Educación, 320, II-38.

Pacios, A. (1959) La Inspección de Enseñanza Media. Revista Bordón, vi, 84-85, 203-213.

Sarasúa, A. (20II) La Inspección, pasado, presente y futuro. Escuela, 3876.

Soler, E.; López del Castillo, M. T.; Muñoz, D.; Molero, A. y Ramírez, E. (1995) Estudios históricos sobre la Inspección Educativa. Madrid: Escuela Española.

Utande, M. (1964) Ley de Ordenación de la enseñanza Media de 26 de febrero de 1953 anotada $y$ comentada. Madrid: Dirección General de Enseñanza Media.

Utande, M. (1975) Treinta años de Enseñanza Media (1938-1968). Revista de Educación, 240, 73-86.

Vera, J. M. (2005) El marco jurídico de la Inspección de Educación. Avances de Supervisión Educativa, I. Recuperado el 20 de marzo de 2015 , de http://www.adide.org/revista/index. php?option $=$ com_content\&task $=$ view $\& i d=41 \&$ Itemid $=3$ I.

Vera, J. M. (2014) Supervisión e Inspección a través del tiempo. Cuadernos de Pedagogía, 44I, 63-66.

VIÑaO, A. (1999) La Inspección Educativa: análisis socio-histórico de una profesión. Revista Bordón, 5I, 3, 251-263. 